\title{
Emotional arousal enhances the impact of long-term memory in attention
}

Article

Accepted Version

Turkileri, N., Field, D. T. ORCID: https://orcid.org/0000-00034041-8404, Ellis, J. A. and Sakaki, M. ORCID:

https://orcid.org/0000-0003-1993-5765 (2021) Emotional arousal enhances the impact of long-term memory in attention. Journal of Cognitive Psychology, 33 (2). pp. 119-132. ISSN 2044-592X doi:

https://doi.org/10.1080/20445911.2021.1883031 Available at https://centaur.reading.ac.uk/95995/

It is advisable to refer to the publisher's version if you intend to cite from the work. See Guidance on citing.

To link to this article DOI: http://dx.doi.org/10.1080/20445911.2021.1883031

Publisher: Taylor \& Francis

All outputs in CentAUR are protected by Intellectual Property Rights law, including copyright law. Copyright and IPR is retained by the creators or other copyright holders. Terms and conditions for use of this material are defined in the End User Agreement.

www.reading.ac.uk/centaur 


\section{CentAUR}

Central Archive at the University of Reading

Reading's research outputs online 
Emotional arousal enhances the impact of long-term memory in attention

Nilgun Turkileri ${ }^{\mathrm{a}^{*}}$, David T. Field ${ }^{\mathrm{b}}$, Judi A. Ellis ${ }^{\mathrm{b}}$, \& Michiko Sakaki $^{\mathrm{bc}}$

a Nevsehir Haci Bektas Veli University, 2000 Evler Mah. Zübeyde Hanim Cad. 50300, Nevsehir, Turkey

b School of Psychology and Clinical Language Sciences, University of Reading, Earley Gate, Whiteknights, Reading RG6 6AL, UK

${ }^{\mathrm{c}}$ Hector Research Institute of Education Sciences and Psychology, University of Tübingen, Europastraße 6, 72072, Tübingen, Germany

* Corresponding author.

E-mail addresses: nilturkileri@gmail.com (N. Turkileri), d.t.field@reading.ac.uk (D. T. Field), j.a.ellis@reading.ac.uk (J. A. Ellis), michiko.sakaki@uni-tuebingen.de (M. Sakaki).

Funding details: This work was supported by grants from the European Commission (PCIG13-GA-2013-618600) and the Japan Society for the Promotion of Science (JP16H05959; JH16H06406). The first author was personally funded by the Republic of Turkey Ministry of National Education in accordance with the law no. 1416. 
AROUSAL AND MEMORY-GUIDED ATTENTION 


\title{
AROUSAL AND MEMORY-GUIDED ATTENTION
}

\begin{abstract}
Research reveals that long-term memory guides attention. However, it remains unclear how it interacts with emotional arousal to guide attention. To address this issue, we asked participants to learn the locations of a target key embedded within scenes (i.e., a training phase). On the next day, participants’ arousal was manipulated by presenting with a previously fear-conditioned tone (CS+) or a neutral tone that had not been paired with electrical stimulation (CS-), followed by the brief presentation of a scene from the training phase. Participants indicated whether the scene included the target key. The target was presented at the same location as in the training phase. Results revealed that $\mathrm{CS}+$, compared with CS-, led to faster target detection, suggesting that arousal induced by CS+ enhanced the effects of long-term memory in guiding attention. These findings support our hypothesis: arousal amplifies the effects of priority in visual search due to long-term memory.
\end{abstract}

Keywords: arousal biased competition; memory-guided attention; spatial memory; visual search 


\section{Introduction}

It is well established that emotionally arousing stimuli, such as snakes, spiders, emotional facial expressions, and erotic pictures, are preferentially processed compared to non-emotional stimuli (for reviews, see Öhman \& Mineka, 2001; Schupp, Flaisch, Stockburger, Jungho, \& Junghöfer, 2006; Vuilleumier \& Pourtois, 2007). Research further suggests that arousal induced by emotional stimuli affects the way we process other stimuli nearby in space or time (Hurlemann et al., 2005; Kensinger, Garoff-Eaton, \& Schacter, 2007; Strange, Hurlemann, \& Dolan, 2003). For example, previous research with rapid serial visual presentation (RSVP) indicates that people show impaired perception of neutral stimuli that are presented shortly after emotional stimuli (A. K. Anderson, 2005; Arnell, Killman, \& Fijavz, 2007; Ihssen \& Keil, 2009; Mathewson, Arnell, \& Mansfield, 2008; Most, Chun, Widders, Haven, \& Zald, 2005; Schwabe et al., 2011; Smith, Most, Newsome, \& Zald, 2006). However, emotional arousal does not always impair processing of the stimuli nearby in space or time (for a review, see Levine \& Edelstein, 2009). Rather, other studies found that exposure to emotionally arousing information sometimes enhances memory (A. K. Anderson, Wais, \& Gabrieli, 2006; Kensinger et al., 2007) and perception of a nearby neutral event (Phelps, Ling, \& Carrasco, 2006; Zeelenberg \& Bocanegra, 2010).

The arousal-biased competition (ABC) theory was proposed to explain these conflicting results (Mather \& Sutherland, 2011). According to the ABC theory, arousal enhances perception and memory of high-priority information, whereas it impairs perception and memory of low-priority information. Priority is determined by stimulus goal-relevance and perceptual saliency (e.g., brightness, contrast). There has been accumulating evidence to support ABC theory-based predictions both for bottom-up saliency (Sutherland \& Mather, 2015, 2018) and for top-down goals (Lee, Greening, \& Mather, 2015; Sakaki, Fryer, \& Mather, 2014). For instance, in one study (Sutherland \& Mather, 2012) when participants 


\section{AROUSAL AND MEMORY-GUIDED ATTENTION}

briefly heard arousing vs. non-arousing sound clips followed by an array of letters, they reported high-salient letters (i.e., dark grey) more than low-salient letters (i.e., light grey) after hearing arousing sounds compared to neutral non-arousing sounds. Recent fMRI research also suggests that emotional arousal has opposing effects on processing of high vs. low salient stimuli (Lee et al., 2018; Lee, Sakaki, Cheng, Velasco, \& Mather, 2014). For instance, in Lee et al. (2018), participants heard a fear-conditioned or a neutral tone, followed by the presentation of a scene and an object image; the activity in the parahippocampal place area was enhanced by the fear-conditioned tone when the scene was more salient than the object, while the activity in the same region was inhibited by the fear-conditioned tone when the scene was less salient than the object. However, previous studies testing the ABC theory primarily focused on the effects of priority signals that come from top-down goals or bottomup saliency. Therefore, it is unclear whether arousal interacts with priority when it is determined by other sources.

Recent theoretical frameworks suggest that stimulus priority is determined not only by top-down goals or bottom-up visual inputs but also by prior experiences; thus, long-term memory is an important factor that determines stimulus priority (for reviews, see Awh, Belopolsky, \& Theeuwes, 2012; Hutchinson \& Turk-Browne, 2012; Peelen \& Kastner, 2014; Theeuwes, 2018, 2019). Although the effects of long-term memory are not purely based on stimulus bottom-up saliency, they are often as fast as attention guided by bottom-up perceptual saliency (Patai, Doallo, \& Nobre, 2012; Rosen, Stern, Michalka, Devaney, \& Somers, 2016; Stokes, 2011; J. J. Summerfield, Lepsien, Gitelman, Mesulam, \& Nobre, 2006; J. J. Summerfield, Rao, Garside, \& Nobre, 2011). In addition, the effects of long-term memory are observed even when they are irrelevant to observers’ current goals (B. A. Anderson, Laurent, \& Yantis, 2011). Thus, recently proposed theories suggest that attention driven by prior knowledge can be a third category of attentional selection in addition to top- 
down and bottom-up attention (B. A. Anderson, 2013; Awh et al., 2012; Failing \& Theeuwes, 2018; Theeuwes, 2019).

Consistent with this view, previous studies demonstrate that prior experiences and expectancies based on memory guide visual attention and modulate cognitive performance, irrespective of whether the experiences are deliberately remembered or automatically activated (Chun \& Jiang, 1998, 2003; Chun \& Turk-Browne, 2007; Henderson \& Hollingworth, 1999; Moores, Laiti, \& Chelazzi, 2003; Olivers, 2011; Rosen et al., 2016; Ryan, Althoff, Whitlow, \& Cohen, 2000; Ryan \& Cohen, 2004; Salvato, Patai, \& Nobre, 2016; Stokes, Atherton, Patai, \& Nobre, 2012; J. J. Summerfield et al., 2006). For instance, Summerfield and colleagues compared performance in the following two tasks: a) detection of a target when the target appeared at the location predicted by memory (i.e., a memory guided attention task) vs. b) detection of a target when the target appeared at the location preceded by a visual spatial cue (i.e., a visual orientation task) (J. J. Summerfield et al., 2006). Their results indicated that the target detection was facilitated not only by the visual spatial cue but also by the long-term memory cue. The facilitative effects of long-term memory were greater than those of visual spatial cues, suggesting the powerful effect of longterm memory on priority and our mental processes.

The present study aimed to investigate the interaction between emotional arousal and long-term memory in guiding attention. As discussed above, past research has shown that emotional arousal modulates the effects of bottom-up saliency (Sutherland \& Mather, 2012, 2015) and top-down goals (Lee et al., 2015; Sakaki et al., 2014). Given that long-term memory can also influence information priority and therefore guide an individual's attention (Theeuwes, 2018, 2019) and given the ABC theory's prediction that arousal has selective enhancement effects on high-priority information (Mather \& Sutherland, 2011), we expected that arousal would enhance detection of a target when it appeared at the location predicted by 
long-term memory. To test this prediction, we used an experimental paradigm adapted from Summerfield et al. (2006). Participants first completed a training phase, where they learned the locations of a target embedded within natural scenes; half of the scenes included the target (the target memory condition), whereas the other half did not include the target (the notarget memory condition). On the following day, participants completed a test phase, during which they were first presented with a tone previously paired with electrical stimulation $(\mathrm{CS}+)$ or a tone that had never been paired with electrical stimulation (CS-). Participants were then shown scenes from the training phase and asked to indicate whether the target was present in each scene. Scenes from the target memory condition should provide predictive information about the location of the target, but those in the no-target memory condition should not provide any information about the location of the target. If arousal enhances attention to high-priority information as the ABC theory suggests, CS+, relative to CS-, should lead to faster reaction times to detect the target in the target memory condition but not in the no-target memory condition.

\section{Materials and Method}

\section{Participants}

Forty-seven undergraduate students at the University of Reading were invited to take part in the study. The study was powered to detect a small-to-medium sized effect (Cohen's f $=.24$ ) based on findings observed in a previous study of the ABC theory (Sakaki et al., 2014). One participant responded prematurely (i.e., before stimulus presentation) in more than $1 / 3$ of the trials during the test phase (see Procedures); data from this participant were excluded before data analyses. Thus, the analyses were conducted on data from 46 participants (38 females, 8 males, $M_{\text {age }}=19.35, \mathrm{SD}=1.49$, age range $=18-24$ ). Acquisition of skin conductance reactions (SCR) failed for four participants due to technical problems. Therefore, SCR analyses did not include these participants' data. In addition, due to 
AROUSAL AND MEMORY-GUIDED ATTENTION

programming errors, participants’ responses were recorded only for scenes in the target memory condition and not for the no-target memory condition for the first six participants during the explicit memory test (see Procedures). This made it difficult for us to compute the correct rejection rate in the task for these six participants, and therefore, the correct rejection rate in the explicit memory test was obtained based on the remaining 40 participants. All participants had normal or corrected-to-normal vision. The study was approved by the University Research Ethics Committee of the University of Reading (UREC 15/17); all participants gave informed written consent and were debriefed accordingly.

\section{Design}

We employed a 2 (memory: target memory, no-target memory) X 2 (arousal: CS+, CS-) within-participant design.

\section{Stimuli}

Stimuli included 96 photographs that depicted indoor scenes (e.g., kitchen; bathrooms) or outdoor landscapes/scenes (e.g., forests). They were obtained from online open sources through the Internet and randomly assigned to one of the conditions for each participant. Additionally, 12 scenes were used in the practice trials. For each scene, three versions were created. In one version, the original scene was presented as a picture of dimension $1000 \times 750$ pixels in 32-bit colour. In the second and third versions, a small yellow key was embedded within the scene. These two versions were identical except for the size of the key: a smaller key (15 x 29 pixels) was embedded in the second version and a bigger key (25 x 49 pixels) was embedded in the third version as done in previous research (J. J. Summerfield et al., 2006). The second version was used in the training phase, while the third version was used in the test phase (see Procedures). The location of the key was determined such that the key would not capture attention through its bottom-up saliency. Specifically, for each scene, we first randomly determined the location of the target key. We next ensured that 
the key was not embedded at one of the three most salient areas in the resulting scene, using the Graph-Based Saliency algorithm (Harel, Koch, \& Perona, 2007) in Matlab R2013a (Mathworks Inc., Sherborn, MA, USA). When the key was detected in one of the three most salient areas in the image, the key location was randomly reallocated and the algorithm was run again. This procedure was repeated until the key was embedded in a non-salient area. We did not control whether the target key was presented in the left and right visual fields (52 pictures with the target key in the left visual field and 44 pictures with the target key in the right visual field).

\section{Procedures}

All participants completed two sessions that took place on two consecutive days.

Session 1. Participants completed the training phase which included six blocks of a visual search task. In each block, they viewed 96 scenes in a randomised order; the order was different across blocks. Half of the 96 scenes included the small yellow key (i.e., the target memory condition) and the other half did not include the key (i.e., the no-target memory condition; see Figure 1A). Participants were instructed to find the key and remember its locations as much as possible. They were also instructed to pay attention to the images that did not include the key.

For each scene, participants first indicated whether they found the key or not by pressing either the left button of a computer mouse (when the key was present) or the right button of the mouse (when they thought the key was absent). The available search time decreased as the blocks progressed in order to make sure all participants spent a roughly similar amount of time (we expected that participants would need less time as the blocks increased). The maximum duration of each scene was randomly determined within a range of 16-24 s in the first block, 12-20 s in blocks 2 and 3, 10-18 s in blocks 4 and 5, and 8-16 s in the last block. Participants were encouraged to respond within these time windows. A similar 
procedure was used in previous studies that employed this paradigm (Patai et al., 2012;

Stokes et al., 2012; J. J. Summerfield et al., 2006). To ensure that participants memorised the location of the target key in each scene; when they pressed the left button, they were asked to drag the mouse to the location of the key and to click on the left button of the mouse again to save their response and to move to the next trial. After a response had been made or after the available search time had expired, participants were presented with a fixation cross for 500 ms, followed by the next trial. Feedback was not provided after each trial in this or the main test phase (which took place in Session 2) because performance feedback can induce emotional responses and may obscure the effects of our manipulation on arousal (Venables \& Fairclough, 2009). Other studies with a similar experimental paradigm (e.g., Summerfield et al., 2006) also did not include feedback.

[Figure 1 near here]

Session 2. On the following day, participants completed a fear-conditioning task, where they learned associations between a tone and electrical stimulation (UCS) delivered to the ring finger of their left hand (see Figure 1B). During each trial, they were presented with one of the two tones (that were different in their pitches) for $700 \mathrm{~ms}$. One of the tones sometimes led to electrical stimulation for $500 \mathrm{~ms}(\mathrm{CS}+)$, whereas the other tone never led to electrical stimulation (CS-). The assignment of the two tones to the CS+/CS- conditions was counterbalanced across participants. There was a 2300-ms inter-stimulus interval (ISI) between the end of the tone and the start of the shock, followed by a fixation cross for $10 \mathrm{~s}$. The participants' task was to press either the right or left arrow key with their right hand to indicate whether the tone was high- or low-pitched irrespective of whether they received a shock or not. There were 30 trials: 10 trials for CS+ with electrical stimulation, 10 trials for CS+ without electrical stimulation, and 10 trials for the CS- condition. Prior to the fearconditioning task, we informed participants which tone would lead to shock but did not tell 


\section{AROUSAL AND MEMORY-GUIDED ATTENTION}

them the probability of the shock (50\%). We also asked participants to rate how uncomfortable they felt about the idea of receiving the shock using a 9-point Likert scale (1: not at all arousing -9: extremely arousing) before the fear-conditioning task (Time 1).

Next, participants completed the test phase (Figure 1C). In each trial, they were first presented with either the CS+ or CS- tone for 700 ms, followed by a 1000-ms fixation cross. Participants were then presented with a scene from the training phase without the target key for 100 ms (“a cue”). After a 1000-ms fixation, they saw the same scene again with or without the target key for 200 ms (“a test image”), followed by a fixation cross. Participants were asked to press the " 1 " key if the test image included the yellow key or to press the " 2 " if the test image did not include the target key. They had to make a response within 2 sec; if they missed it, the fixation cross automatically disappeared and participants saw a warning instruction to respond faster. The next trial started after a jittered 2-8 s interval. When participants heard the CS+ tone, in the majority of the trials, they did not receive stimulation. To prevent extinction, participants also received stimulation in 16 trials in total (i.e., booster trials). In these booster trials, electric shock was administered to their ring finger right after the second presentation of the scene, which was followed by a longer inter-trial interval (10 sec). Participants were asked to press either the " 1 " or " 2 " key to indicate whether the test image included the target or not even in these booster trials.

The test phase included three blocks, each of which included 32 trials (96 trials in total). The test image included the target on two-thirds of the trials (64 trials; key present trials). We also included trials where the test image did not include the key as catch trials to ensure that participants engaged with the task (32 trials; key absent trials). Among the 64 key-present trials, 32 trials included scenes from the target memory condition (see Figure 1A1; 13 trials with CS+, 13 trials with CS-, and 6 booster trials) and the other 32 trials 
AROUSAL AND MEMORY-GUIDED ATTENTION

included scenes from the no-target memory condition (see Figure 1-A2 and Table 1; 14 trials with CS+, 13 trials with CS-, and 5 booster trials ${ }^{1}$ ).

[Table 1 near here]

After the test phase, participants rated how much they felt uncomfortable when they received the shock using the same 9-point Likert scale as Time 1 (Time 2). Next, we assessed participants' explicit memory for the location of the target key. In this explicit memory test, a trial began with the presentation of a scene without the target key. Participants indicated whether the scene had included the key or not based on their memory. When they decided that the scene had included the key, they were asked to press the left button of the mouse and the target key appeared at the centre of the screen. Then, they were asked to drag and move the key to the location where they thought it had been before. In contrast, when they decided that the scene had not include the key, they pressed the right button of the mouse and moved to the next trial. Feedback indicating whether the response was correct or incorrect was provided after participants made the initial decision in each trial. The duration of the trials was self-paced and there was no time limit.

\section{Apparatus}

The stimuli were presented on a screen (resolution of 1680 x 1050 pixel) using Psychtoolbox (Brainard, 1997; Pelli, 1997) in Matlab R2013a (The Mathworks Corp. Natrick, MA). Administration of the electrical stimulation was performed using the Powerlab system and Labchart version 7 (ADInstruments Ltd, Chalgrove, Oxfordshire, UK). During the fear-conditioning task, participants' SCR were also recorded from the middle and index fingers of their left hand at $1000 \mathrm{~Hz}$ sampling rates.

\section{Electric shock}

\footnotetext{
${ }^{1}$ Due to programming errors, we had an unequal number of trials across conditions for all participants: 13 trials with CS+ for the target memory condition but 14 trials with CS+ for the no-target condition (see Table 1).
} 
Prior to Session 2, we determined the amplitude and frequency of a "highly unpleasant but not painful” level of electrical stimulation for each participant $(M=3.91 \mathrm{~mA}$, $\mathrm{SD}=2.22 ; M=52.41 \mathrm{~Hz}, \mathrm{SD}=42.68)$. The level determined was used throughout the fearconditioning phase. To prevent habituation to the shock, after the fear-conditioning task and after each block of the test phase, we asked participants whether they still felt "highly unpleasant but not painful” feelings to the shock. Some participants $(N=14)$ asked the experimenters to slightly increase the level of stimulation to make the shock "highly unpleasant but not painful” and therefore, we increased the level of stimulation for this subset of participants (the averaged increase of shock was $0.46 \mathrm{~mA}$ and $9.33 \mathrm{~Hz}$ ). Four participants rather asked the experimenters to decrease the level of stimulation because they felt too uncomfortable with the initial level and therefore we adjusted the level of stimulation for this small number of participants (the average change was $0.25 \mathrm{~mA}$ and $5.35 \mathrm{~Hz}$ ). This procedure ensured that the administration of stimulation was subjectively felt "unpleasant but not painful” for all participants throughout the experimental session.

\section{SCR analysis}

SCR data epochs were extracted from a time window between 0 and $10 \mathrm{~s}$ after CS tone onset, and baseline-corrected between 0 and $1 \mathrm{~s}$. The peak SCR amplitude was taken between 2 and 10 s from the trial-by-trial average SCR epoch as a function of CS tone. Data from the first trial were removed as responses in this trial could reflect orientation response (Li et al., 2017).

\section{Results}

Results from booster trials were excluded from all analyses (both the fearconditioning and the test phases) because we were interested in the effects of emotional arousal induced by the anticipation of shocks, rather than the effect of the shock per se, which was a similar procedure to the previous studies (e.g., Lee et al., 2014). 


\section{AROUSAL AND MEMORY-GUIDED ATTENTION}

\section{Training phase}

Reaction times and accuracy during the training phase were analysed with linear mixed-effect modelling with block as a predictor to examine whether participants' performance improved across blocks with the statistical package SPSS 25 for Mac OS. The model also included the random participant intercepts and the random participant slope for blocks. The analysis on accuracy revealed a significant effect of block, beta $=.74, \mathrm{SE}=.16, t$ $(45)=4.61, p<.001,95 \%$ CI $[0.42,1.07]$; suggesting that there is a significant positive relationship between blocks and performance accuracy. A similar analysis on reaction times also revealed a significant effect of block, beta $=-.66, \mathrm{SE}=.04, t(45)=-15.40, p<.001$, 95\% CI [-0.75, -0.57]; reflecting that reaction times significantly decreased over six blocks (Figure 2A). The percentage of scenes where participants correctly identified the target key $(M=97.56 \%, \mathrm{SD}=1.18)$ and the percentage of scenes where they correctly identified the absence of the target $(M=94.25 \%$, SD $=1.31)$ were also above $90 \%$ across all blocks. These results indicate that participants successfully learned the target's location in each scene as observed in other studies with a similar paradigm (Patai et al., 2012; Raeder et al., 2019; Stokes et al., 2012; J. J. Summerfield et al., 2006)

[Figure 2 near here]

\section{Fear-conditioning phase}

SCRs were greater in the CS+ trials $(M=0.16$ microS, $S D=0.19)$ than in the CStrials $(M=0.12$ microS, $S D=0.15$; Figure 2B), $t(41)=1.96, p=.05, d=0.42,95 \%$ CI [0.001, 0.12]. Thus, participants appeared to acquire a fear response to the CS+ tone, though the difference did not reach statistical significance. We also found that participants reported stronger negative feelings towards electric shock at Time $2(M=4.33, \mathrm{SD}=1.51)$ relative to Time $1(M=3.27, \mathrm{SD}=1.72), t(41)=3.35, p<.01, d_{z}=.66,95 \%$ CI $[.42,1.71]$, suggesting 
AROUSAL AND MEMORY-GUIDED ATTENTION

that participants maintained their negative feelings towards shock over the course of the experiment.

\section{Performance in the test phase}

Given that our main focus was on the effects of emotional arousal on the target detection based on long-term memory, our analyses focused on performance in the key present trials in the test phase. The average accuracy of participants' responses in the catch trials (i.e., the key absent trials) was high $(M=88.76 \%, S D=31.59)$, confirming that participants engaged with the task. For the key-present trials, trials with scene images where the participant failed to find the target key on more than $50 \%$ of trials in the training phase were excluded from the analyses reported in this section (1.39\% of all trials) to allow us to examine the effects of emotion on visual search based on long-term memory. ${ }^{2}$

The mean reaction times from trials where participants made correct responses during the test phase were obtained for each participant for each condition. A repeated-measures analysis of variance (ANOVA) with factors arousal (CS+ or CS-) and memory condition (target memory or no-target memory) was performed on these mean reaction times using SAS Proc Mixed procedures (SAS 9.4; SAS Institute, Raleigh, NC, USA). This analysis showed a significant interaction between memory and arousal (Figure 3), $F(1,44)=10.18, p$ $<.005, \eta_{\mathrm{p}}{ }^{2}=.25$, with no significant main effects ( $p s>.10$ ). Subsequent simple effect tests revealed that in the target memory condition, participants were faster to identify the target key in the CS+ condition $(M=505.99 \mathrm{~ms}, S D=268.45)$ than in the CS- condition $(M=593.54$ ms, $S D=275.15), t(44)=-2.71, p<.01, d_{z}=.40,95 \%$ CI [-152.48 -22.63]. In contrast, the CS+ vs. CS- conditions were not significantly different in the no-target memory condition ( $p>$ .10). Viewing the interaction differently, when participants were presented with the CS+ tone, they were faster to identify the target key in the target memory trials ( $M=505.98 \mathrm{~ms}$,

\footnotetext{
${ }^{2}$ One participant did not have any correct responses in the CS+/no-target memory condition during the test phase. Data from this participant were still included in our analyses but performance for the condition was treated as missing data in the analysis on the reaction times.
} 
$S D=268.45)$ compared to the no-target memory trials $(M=592.24 \mathrm{~ms}, S D=281.76), t(44)=$ 3.47, $p<.005, d_{z}=.52,95 \%$ CI [36.09 136.42]. In contrast, the difference between the two memory conditions was not significant in CS- trials $\left(M_{\text {target-memory }}=593.54 \mathrm{~ms}\right.$ vs. $M_{\text {no-target- }}$ memory $=541.76 \mathrm{~ms} ; p=.071)$. These results are consistent with our hypothesis and suggest that emotional arousal induced by CS+ facilitates the target detection based on long-term memory.

[Figure 3 near here]

To investigate performance accuracy, a similar 2 (arousal) x 2 (memory) repeatedmeasures ANOVA was performed on the average accuracy levels. This analysis revealed a significant main effect of memory, $F(1,45)=104.22, p<.001, \eta_{\mathrm{p}}{ }^{2}=.72$, reflecting that participants were more likely to find the target key in the target memory condition $(M=$ $75.23 \%, S E=2.19)$ compared to no-target memory condition $(M=49.10 \%, S E=2.19)$. There was also a main effect of arousal, $F(1,45)=6.22, p<.05, \eta_{\mathrm{p}}{ }^{2}=.12$, reflecting that participants were better at finding the target key in the CS+ condition $(M=64.19 \%, S E=$ 1.95) compared to the CS- condition $(M=60.14 \%, S E=1.95)$. However, the interaction effect between arousal and memory was not significant $(p>.50)$. Therefore, the arousal-bymemory interaction observed in the reaction times cannot be simply explained by the speedaccuracy trade-off (Reed, 1973). We also found that participants' accuracy was significantly above chance in the target memory conditions (Table 2): CS+/target memory, $t(45)=12.80, p$ $<.001$; CS-/target memory, $t(45)=10.38, p<.001$. However, accuracy performance in the no-target memory conditions were below chance level (CS+/no-target memory: $p=.79$ and CS-/no-target memory: $p=.36)$.

[Table 2 near here]

\section{Explicit memory test}

As described above, our results from the test phase suggest that CS+ facilitated the 
detection of the target key when it appeared at the location predicted by long-term memory relative to the CS- condition. To address whether this significant difference was due to the fact that participants had better memory for the target location for scenes presented in the CS+ condition than in the CS- condition by chance, we analysed performance in the explicit memory test. During this task, participants correctly identified whether or not the scene included the target in $61.16 \%$ of trials in the target memory condition (i.e., hit rates) and 93.57\% of trials in the no-target memory condition (i.e., correct rejection rates).

For the scenes which the participant correctly identified, and which it included the target key in the training session, we calculated the Euclidean distance between a) the original location of the target key learned in the training session and b) the location that the participants indicated in the explicit memory test. This distance measure did not show a significant difference between CS+ $(M=108.55$ pixels, $S D=90.09)$ and CS- $(M=104.67$ pixels, $S D=75.13)$ trials $(p=.68)$. Thus, results from the test phase were not readily explained by the difference in memory strength between those used in the CS+ condition than those used in the CS- condition. Hit rates were also similar in two conditions (60.76\% for the CS+ condition and $61.31 \%$ for the CS- condition) and did not differ than each other $(p=.81)$. We also found that participants identified which scenes included the target significantly above chance both in the CS+ and CS- conditions, $t(45)=3.50, p<.01,95 \%$ CI [4.58 16.96]; $t(45)=3.92, p<.001,95 \%$ CI [5.50 17.11], respectively.

We next conducted a 2 (arousal: CS+ or CS-) x 2 (explicit memory accuracy: correct or incorrect) repeated-measures ANOVA on the mean reaction times in the test phase in order to examine whether explicit memory interacts with arousal to affect target detection. Trials from the no-target memory condition were not included in this analysis because we do not have explicit memory measures for these scenes. This analysis confirmed a significant main effect of arousal, $F(1,45)=8.91, p<.01, \eta_{\mathrm{p}}^{2}=.16$, indicating faster reaction times to detect 
the target in the CS+ condition $(M=582.26, S E=41.09)$ compared to the CS- condition $(M=$ 686.11, $S E=55.58)$. Neither a main effect of explicit memory accuracy nor an interaction between arousal and explicit memory accuracy was significant ( $p s>$.30). These results suggest that arousal has similar facilitative effects on the target detection irrespective of whether individuals have correct or incorrect memories of the target location.

\section{Discussion}

According to the ABC theory (Mather \& Sutherland, 2011), emotional arousal favours processing of high-priority information over low-priority information. While previous studies on the ABC theory predominantly focused on the priority signals determined by goal-relevance or bottom-up saliency (Lee et al., 2015, 2018, 2014; Sakaki et al., 2014; Sutherland \& Mather, 2012, 2015), an increasing number of studies suggest that individuals’ long-term memory is also a critical factor affecting the priority of information (Patai et al., 2012; Stokes et al., 2012; J. J. Summerfield et al., 2006, 2011). In fact, recent accounts of selective attention suggest that attention is often driven by past episodes beyond a dichotomous mechanism of stimulus bottom-up saliency or top-down goals/intentions (Awh et al., 2012; Theeuwes, 2019). In the present study, we tested whether emotional arousal enhances processing of stimuli that have high-priority based on long-term memory, by presenting a fear-conditioned (CS+) or a neutral tone (CS-) during the memory-guided attention paradigm used in previous research (e.g., J. J. Summerfield et al., 2006).

We found that the detection of the target key was facilitated by the CS+ tone more than the CS- tone when the target key was presented at the location predicted by prior memory. In contrast, the facilitation effects were not observed when participants did not know where to expect the target key, and thus there were no clear priority signals for the target (the no-target memory condition). Participants’ arousal levels measured by SCR yielded greater responses to CS+ cues compared with CS- cues. Although this difference was 


\section{AROUSAL AND MEMORY-GUIDED ATTENTION}

only approaching significance, participants reported significantly more negative feelings towards electric shock after the experiment than before. Furthermore, during the test phase, the detection of the target key was significantly altered by the CS+ tone as described above. These results suggest that participants acquired and maintained fear responses to the CS+ tone during the experiment; and that arousal induced by the CS+ tone affected the detection of the target. Our results are consistent with our prediction that arousal not only interacts with the priority signals determined by top-down goals or bottom-up saliency but also with the priority signals determined by long-term memory.

The human visual system is highly sensitive to familiarity in the environment so that the brain can prioritize important information and use its resources accordingly (Chun \& Jiang, 2003; Rosenbaum \& Jiang, 2013). Thus, long-term memories allow the brain to distinguish between old and new information to form representations of the environment. Places or objects that were previously important are often prioritised during visual search, even though observers may not be aware of the previous exposures (e.g., Jiang, Swallow, \& Rosenbaum, 2013). Locations that were known in the past will preserve their importance in the future, reducing the "computational burden" and, thus, helping guide visual search (C. Summerfield \& Egner, 2009). Past experiences serve as a basis of our expectation in a given environment. Emotional arousal is also known to enhance prioritisation processes (Mather \& Sutherland, 2011). Therefore, it is reasonable to expect that arousal also enhances attending to a previously learnt location, possibly by reducing the number of competitors for representation in a given scene and at a given time. Consistent with the above literature, the results from the current study suggest that emotional arousal amplifies the effects of longterm memory in guiding attention.

Previous research investigating emotional effects on memory (e.g., Christianson, Loftus, Hoffman, \& Loftus, 1991; Kensinger et al., 2007) and attention (e.g., Eastwood, 
Smilek, \& Merikle, 2001; Fox, Russo, \& Dutton, 2002; Öhman, Flykt, \& Esteves, 2001) has often used negative visual stimuli (such as images of snakes, spiders, and fearful faces). A recent study examined the effects of these negative stimuli on memory-guided attention (Raeder et al., 2019). Using a similar paradigm to ours, Raeder and colleagues had participants learn the location of a small key (a target) embedded within a scene image during the learning phase. Participants completed a test phase 24 hours later, during which they were first shown scenes they had seen during the learning phase without the target (a cue image), followed by the same scenes with the target presented either at the learned location or a novel location (a test image). Participants' task was to detect the target in the test images as in our study. Critically, the researchers manipulated the cue images so that they included either a fearful or a neutral face (shown at different locations from the subsequent target key). Their results showed that the presentation of fearful faces impaired the participants' ability to identify the target in the subsequent test image. These results may appear contradictory to the $\mathrm{ABC}$ theory and our results that emotional arousal enhances the effects of priority due to long-term memory.

However, in Raeder et al. (2019), the presentation of fearful faces impaired the target detection irrespective of whether the target was shown at the learned location or not. Thus, the impairing effects were general - not specific to a condition where long-term memory is expected to facilitate performance. In addition, in their study, emotional stimuli were presented at the same time in the same modality as the cue image. Thus, it is likely that the fearful face and the cue image were competing for limited resources. In contrast, we manipulated participants' arousal level using a fear-conditioned stimulus presented before the cue presentation. The fear-conditioned stimulus was also presented in a different modality from the cue and test images to reduce direct competition between the fear-conditioned stimulus and the visual target (see also Zeelenberg \& Bocanegra, 2010). Thus, our results 
AROUSAL AND MEMORY-GUIDED ATTENTION

extend previous findings and suggest that when emotional stimuli and cues for long-term memory are not in direct competition, arousal induced by the emotional stimuli facilitates memory-guided attention.

One interesting result from this study is that the effects of arousal were similar irrespective of explicit memory test performance. During our experiment, we tested participants' explicit memory about the location of the target for each scene and examined whether the CS+ tone has similar facilitative effects on the target detection irrespective of performance in the explicit memory test. In this analysis, we found that CS+ had similar impacts on the target search irrespective of whether participants correctly retrieved the location of the target key or not. Together with the fact that the cues were presented only for a very short time (100 ms) in the test phase, our results suggest that the effects of arousal on the memory-guiding cues do not necessarily rely on explicit memories. Previous research has also demonstrated that prior experiences and expectancies based on memory guide visual attention and modulate cognitive performance, irrespective of whether the experiences are deliberately remembered or automatically activated (e.g., Chun \& Jiang, 1998, 1999, 2003; Henderson \& Hollingworth, 1999; Jiang et al., 2013; Moores et al., 2003; Olivers, 2011; Rosen et al., 2016; Ryan et al., 2000; Ryan \& Cohen, 2004). For example, Salvato et al. (2016) found that older adults who showed impaired performance in an explicit retrieval task still showed preserved performance in a memory-guided attention task, suggesting that attending to a previously known location does not necessarily rely on explicit recall. Our results extend these prior findings and suggest that emotional arousal modulates the effects of long-term memory even when the memory is not deliberately remembered.

However, in our study, the number of trials per cell was small in this analysis. In addition, we did not experimentally manipulate whether participants explicitly vs. implicitly remembered the location of the target key during the test phase. Thus, the present study does 


\section{AROUSAL AND MEMORY-GUIDED ATTENTION}

not provide a clear answer about whether arousal equally enhances the effects of explicit memory (i.e., memories that participants are consciously aware of) and implicit memory (i.e., memories that participants are not necessarily consciously aware of). Future research should address whether emotional arousal has similar or different impacts on the effects of memory cues depending on whether individuals have explicit vs. implicit memory. Such future research may want to manipulate the cue presentation duration in the test phase given that a cue presented for a longer duration should allow participants to use explicit memory more than the one presented for a short duration (as done in our study).

Another related question for future research concerns whether emotional arousal has similar or different impacts on memory cues depending on the strength of associated memory. When associations between cues and to-be-retrieved memories are relatively weak, individuals may need to exert deliberate efforts to retrieve associated memories when they are presented with cues. This deliberate process would be related to the prefrontal cortex and the executive function (Dobbins, Foley, Schacter, \& Wagner, 2002), which can be impaired by emotional arousal (Kuhbandner \& Zehetleitner, 2011). Thus, emotional arousal may reduce the facilitative effects of memory cues in this context. In contrast, when the links between cues and to-be-retrieved memories are strongly formed after repeated exposure (as done in this study), associated memories can be retrieved automatically and rapidly with few cognitive resources (McDaniel \& Einstein, 2000). We foresee that emotional arousal should not impair this automatic implicit retrieval process. Rather, once memories are retrieved, emotional arousal may amplify the effects of these memories in guiding one's behaviour via the selective enhancement effects on high-priority information. Future research should explicitly manipulate the strength of association to test these predictions.

There are also other questions that remain for future research. First, in the present study, we did not find significant effects of emotional arousal in the no-target memory 
AROUSAL AND MEMORY-GUIDED ATTENTION

condition. According to the ABC theory, emotional arousal should impair attention to lowpriority information (Mather \& Sutherland, 2011). One prediction that can be made is therefore that arousal should impair the target detection in the no-target memory condition because the location of the target key has lower priority in this condition compared with the target memory condition. This lack of significant effects may be due to the lack of a sufficient statistical power. In fact, one of the pairwise tests was approaching significant (see Results). Another possibility is that it was hard for participants to identify the target in these no-target memory trials (where participants did not know where to expect the target key). In fact, while the presentation of the test image was determined based on prior studies (e.g., Patai et al., 2012; Stokes, 2011; J. J. Summerfield et al., 2006), it was relatively short (200 ms). In addition, performance accuracy in the no-target memory trials was not significantly different from chance (see Results). Alternatively, in the present study, we used the same target key across all trials and therefore the target key itself may have gained goal-relevance over the course of the experiment, which may have obscured the impairing effects of arousal on long-term memory. Future research with a larger sample size and a longer presentation duration for the test image is required to test if arousal has impairing effects on attention when individuals do not have a salient memory about the target location. It may be useful in these studies to manipulate the target itself to prevent the same target from being used so many times and thus acquiring high priority. Such future research may alternatively manipulate the priority of the target, using familiar (i.e., prioritised) vs. novel (i.e., not prioritised) locations (Raeder et al., 2019; Salvato et al., 2016).

Second, we did not compare the effects of arousal on the memory-guided attention task vs. the visually guided attention, where visual search was enhanced by a salient visual cue (e.g., J. J. Summerfield et al., 2006). Therefore, it is unclear whether the interaction 


\section{AROUSAL AND MEMORY-GUIDED ATTENTION}

between emotional arousal and memory-guided attention are smaller or larger than those with perceptual saliency documented in the literature.

In summary, this study provides evidence for the role of arousal in memory-guided attention. Our findings suggest that emotional arousal interacts with prior knowledge and one’s expectations to guide current attention, supporting the ABC framework (Mather \& Sutherland, 2011). More generally, the results indicate that emotion makes us even more likely to be biased by the information that has once been prioritised based on memory. 


\section{Declaration of interest statement:}

The authors report no conflict of interest.

\section{Data availability statement:}

The data, stimuli, and the experimental files that support the findings of this study are openly available in OSF at http://doi.org/10.17605/OSF.IO/2B4M7. 


\section{References}

Anderson, A. K. (2005). Affective influences on the attentional dynamics supporting awareness. Journal of Experimental Psychology: General, 134(2), 258-281. https://doi.org/10.1037/0096-3445.134.2.258

Anderson, A. K., Wais, P. E., \& Gabrieli, J. D. E. (2006). Emotion enhances remembrance of neutral events past. Proceedings of the National Academy of Sciences of the United States of America, 103(5), 1599-1604. https://doi.org/10.1073/pnas.0506308103

Anderson, B. A. (2013). A value-driven mechanism of attentional selection stimulus value. Journal of Vision, 13(3):(7), 1-16. https://doi.org/10.1167/13.3.7.doi

Anderson, B. A., Laurent, P. A., \& Yantis, S. (2011). Value-driven attentional capture. Proceedings of the National Academy of Sciences, 108(25), 10367-10371. https://doi.org/10.1073/PNAS.1104047108

Arnell, K. M., Killman, K. V., \& Fijavz, D. (2007). Blinded by emotion: Target misses follow attention capture by arousing distractors in RSVP. Emotion, 7(3), 465-477. https://doi.org/10.1037/1528-3542.7.3.465

Awh, E., Belopolsky, A. V., \& Theeuwes, J. (2012). Top-down versus bottom-up attentional control: a failed theoretical dichotomy. Trends in Cognitive Sciences, 16(8), 437-443. https://doi.org/10.1016/j.tics.2012.06.010

Brainard, D. H. (1997). The Psychophysics Toolbox. Spatial Vision, 10(4), 433-436. https://doi.org/10.1163/156856897X00357

Christianson, S., Loftus, E. F., Hoffman, H., \& Loftus, G. R. (1991). Eye fixations and memory for emotional events. Journal of Experimental Psychology. Learning, Memory, and Cognition, 17(4), 693-701. https://doi.org/10.1037/0278-7393.17.4.693

Chun, M. M., \& Jiang, Y. (1998). Contextual cueing: Implicit learning and memory of visual context guides spatial attention. Cognitive Psychology, 36(1), 28-71.

https://doi.org/10.1006/cogp.1998.0681

Chun, M. M., \& Jiang, Y. (1999). Top-down attentional guidance based on implicit learning of visual covariation. Psychological Science, 10(4), 360-365.

https://doi.org/10.1111/1467-9280.00168

Chun, M. M., \& Jiang, Y. (2003). Implicit, long-term spatial contextual memory. Journal of Experimental Psychology: Learning, Memory, and Cognition, 29(2), 224-234. https://doi.org/10.1037/0278-7393.29.2.224

Chun, M. M., \& Turk-Browne, N. B. (2007). Interactions between attention and memory. Current Opinion in Neurobiology, 17(2), 177-184.

https://doi.org/10.1016/j.conb.2007.03.005

Dobbins, I. G., Foley, H., Schacter, D. L., \& Wagner, A. D. (2002). Executive Control during Episodic Retrieval. Neuron, 35(5), 989-996. https://doi.org/10.1016/s08966273(02)00858-9 
Eastwood, J. D., Smilek, D., \& Merikle, P. M. (2001). Differential attentional guidance by unattended faces expressing positive and negative emotion. Perception \& Psychophysics, 63(6), 1004-1013. https://doi.org/10.3758/BF03194519

Failing, M., \& Theeuwes, J. (2018). Selection history: How reward modulates selectivity of visual attention. Psychonomic Bulletin and Review, 25(2), 514-538. https://doi.org/10.3758/s13423-017-1380-y

Fox, E., Russo, R., \& Dutton, K. (2002). Attentional bias for threat: Evidence for delayed disengagement from emotional faces. Cognition \& Emotion, 16(3), 355-379. https://doi.org/10.1080/02699930143000527

Harel, J., Koch, C., \& Perona, P. (2007). Graph-based visual saliency. Advances in Neural Information Processing Systems, 545-552. Retrieved from http://papers.nips.cc/paper/3095-graph-based-visual-saliency.pdf

Henderson, J. M., \& Hollingworth, A. (1999). High-level scene perception. Annual Review of Psychology, 50, 243-271. https://doi.org/10.1146/annurev.psych.50.1.243

Hurlemann, R., Hawellek, B., Matusch, A., Kolsch, H., Wollersen, H., Madea, B., ... Dolan, R. J. (2005). Noradrenergic modulation of emotion-induced forgetting and remembering. The Journal of Neuroscience, 25(27), 6343-6349. https://doi.org/10.1523/JNEUROSCI.0228-05.2005

Hutchinson, J. B., \& Turk-Browne, N. B. (2012). Memory-guided attention: Control from multiple memory systems. Trends in Cognitive Sciences, 16(12), 576-579. https://doi.org/10.1016/j.tics.2012.10.003

Ihssen, N., \& Keil, A. (2009). The costs and benefits of processing emotional stimuli during rapid serial visual presentation. Cognition and Emotion, 23(2), 296-326. https://doi.org/10.1080/02699930801987504

Jiang, Y. V, Swallow, K. M., \& Rosenbaum, G. M. (2013). Guidance of spatial attention by incidental learning and endogenous cuing. Journal of Experimental Psychology: Human Perception and Performance, 39(1), 285-297. https://doi.org/10.1037/a0028022

Kensinger, E. A., Garoff-Eaton, R. J., \& Schacter, D. L. (2007). How negative emotion enhances the visual specificity of a memory. Journal of Cognitive Neuroscience, 19(11), 1872-1887. https://doi.org/10.1162/jocn.2007.19.11.1872

Kuhbandner, C., \& Zehetleitner, M. (2011). Dissociable effects of valence and arousal in adaptive executive control. PLoS ONE, 6(12). https://doi.org/10.1371/journal.pone.0029287

Lee, T. H., Greening, S. G., \& Mather, M. (2015). Encoding of goal-relevant stimuli is strengthened by emotional arousal in memory. Frontiers in Psychology, 6:1173. https://doi.org/10.3389/fpsyg.2015.01173

Lee, T. H., Greening, S. G., Ueno, T., Clewett, D., Ponzio, A., Sakaki, M., \& Mather, M. (2018). Arousal increases neural gain via the locus coeruleus-noradrenaline system in younger adults but not in older adults. Nature Human Behaviour, 2(5), 356-366. https://doi.org/10.1038/s41562-018-0344-1 
Lee, T. H., Sakaki, M., Cheng, R., Velasco, R., \& Mather, M. (2014). Emotional arousal amplifies the effects of biased competition in the brain. Social Cognitive and Affective Neuroscience, 9(12), 2067-2077. https://doi.org/10.1093/scan/nsu015

Levine, L. J., \& Edelstein, R. S. (2009). Emotion and memory narrowing: A review and goalrelevance approach. Cognition and Emotion, 23(5), 833-875. https://doi.org/10.1080/02699930902738863

Li, J., Chen, W., Caoyang, J., Wu, W., Jie, J., Xu, L., \& Zheng, X. (2017). Moderate partially reduplicated conditioned stimuli as retrieval cue can increase effect on preventing relapse of fear to compound stimuli. Frontiers in Human Neuroscience, 11:575. https://doi.org/10.3389/fnhum.2017.00575

Mather, M., \& Sutherland, M. R. (2011). Arousal-biased competition in perception and memory. Perspectives on Psychological Science, 6(2), 114-133. https://doi.org/10.1177/1745691611400234

Mathewson, K. J., Arnell, K. M., \& Mansfield, C. A. (2008). Capturing and holding attention: The impact of emotional words in rapid serial visual presentation. Memory and Cognition, 36(1), 182-200. https://doi.org/10.3758/MC.36.1.182

McDaniel, M. A., \& Einstein, G. O. (2000). Strategic and Automatic Processes in Prospective Memory Retrieval: A Multiprocess Framework. Applied Cognitive Psychology, 14(7), S127-S144. https://doi.org/10.1002/acp.775

Moores, E., Laiti, L., \& Chelazzi, L. (2003). Associative knowledge controls deployment of visual selective attention. Nature Neuroscience, 6(2), 182-189. https://doi.org/10.1038/nn996

Most, S. B., Chun, M. M., Widders, D. M., Haven, N., \& Zald, D. H. (2005). Attentional rubbernecking: Cognitive control and personality in emotion-induced blindness. Psychonomic Bulletin and Review, 12(4), 654-661. https://doi.org/10.3758/BF03196754

Öhman, A., Flykt, A., \& Esteves, F. (2001). Emotion drives attention: Detecting the snake in the grass. Journal of Experimental Psychology: General, 130(3), 466-478. https://doi.org/10.1037//0096-3445.130.3.466

Öhman, A., \& Mineka, S. (2001). Fears, phobias, and preparedness: Toward an evolved module of fear and fear learning. Psychological Review, 108(3), 483-522. https://doi.org/10.1037/0033-295X.108.3.483

Olivers, C. N. L. (2011). Long-term visual associations affect attentional guidance. Acta Psychologica, 137(2), 243-247. https://doi.org/10.1016/j.actpsy.2010.07.001

Patai, E. Z., Doallo, S., \& Nobre, A. C. (2012). Long-term memories bias sensitivity and target selection in complex scenes. Journal of Cognitive Neuroscience, 24(12), 22812291. https://doi.org/10.1162/jocn_a_00294

Peelen, M. V., \& Kastner, S. (2014). Attention in the real world: Toward understanding its neural basis. Trends in Cognitive Sciences, 18(5), 242-250.

https://doi.org/10.1016/j.tics.2014.02.004 
Pelli, D. G. (1997). The VideoToolbox software for visual psychophysics: Transforming numbers into movies. Spatial Vision, 10(4), 437-442. https://doi.org/10.1163/156856897X00366

Phelps, E. A., Ling, S., \& Carrasco, M. (2006). Emotion facilitates perception and potentiates the perceptual benefits of attention. Psychological Science, 17(4), 292-299. https://doi.org/10.1111/j.1467-9280.2006.01701.x

Raeder, S. M., Bone, J. K., Patai, E. Z., Holmes, E. A., Nobre, A. C., \& Murphy, S. E. (2019). Emotional distraction in the context of memory-based orienting of attention. Emotion, 19(8), 1366-1376. https://doi.org/10.1037/emo0000506

Reed, A. V. (1973). Speed-accuracy trade-off in recognition memory. Science, 181(4099), 574-576. https://doi.org/10.1126/science.181.4099.574

Rosen, M. L., Stern, C. E., Michalka, S. W., Devaney, K. J., \& Somers, D. C. (2016). Cognitive control network contributions to memory-guided visual attention. Cerebral Cortex, 26(5), 2059-2073. https://doi.org/10.1093/cercor/bhv028

Rosenbaum, G. M., \& Jiang, Y. V. (2013). Interaction between scene-based and array-based contextual cueing. Attention, Perception, and Psychophysics, 75(5), 888-899. https://doi.org/10.3758/s13414-013-0446-9

Ryan, J. D., Althoff, R. R., Whitlow, S., \& Cohen, N. J. (2000). Amnesia is a deficit in relational memory. Psychological Science, 11(6), 454-461. https://doi.org/10.1111/1467-9280.00288

Ryan, J. D., \& Cohen, N. J. (2004). Processing and short-term retention of relational information in amnesia. Neuropsychologia, 42(4), 497-511. https://doi.org/10.1016/j.neuropsychologia.2003.08.011

Sakaki, M., Fryer, K., \& Mather, M. (2014). Emotion strengthens high-priority memory traces but weakens low-priority memory traces. Psychological Science, 25(2), 387-395. https://doi.org/10.1177/0956797613504784

Salvato, G., Patai, E. Z., \& Nobre, A. C. (2016). Preserved memory-based orienting of attention with impaired explicit memory in healthy ageing. Cortex, 74, 67-78. https://doi.org/10.1016/j.cortex.2015.10.019

Schupp, H. T., Flaisch, T., Stockburger, J., Jungho, M., \& Junghöfer, M. (2006). Emotion and attention: event-related brain potential studies. Progress in Brain Research, 156, 31-51. https://doi.org/10.1016/S0079-6123(06)56002-9

Schwabe, L., Merz, C. J., Walter, B., Vaitl, D., Wolf, O. T., \& Stark, R. (2011). Emotional modulation of the attentional blink: The neural structures involved in capturing and holding attention. Neuropsychologia, 49(3), 416-425.

https://doi.org/10.1016/j.neuropsychologia.2010.12.037

Smith, S. D., Most, S. B., Newsome, L. A., \& Zald, D. H. (2006). An emotion-induced attentional blink elicited by aversively conditioned stimuli. Emotion, 6(3), 523-527. https://doi.org/10.1037/1528-3542.6.3.523 
Stokes, M. G. (2011). Top-down visual activity underlying VSTM and preparatory attention. Neuropsychologia, 49(6), 1425-1427. https://doi.org/10.1016/j.neuropsychologia.2011.02.004

Stokes, M. G., Atherton, K., Patai, E. Z., \& Nobre, A. C. (2012). Long-term memory prepares neural activity for perception. Proceedings of the National Academy of Sciences of the United States of America, 109(6), 360-367. https://doi.org/10.1073/pnas.1108555108

Strange, B. A., Hurlemann, R., \& Dolan, R. J. (2003). An emotion-induced retrograde amnesia in humans is amygdala- and beta-adrenergic-dependent. Proceedings of the National Academy of Sciences of the United States of America, 100(23), 13626-13631. https://doi.org/10.1073/pnas.1635116100

Summerfield, C., \& Egner, T. (2009). Expectation (and attention) in visual cognition. Trends in Cognitive Sciences, 13(9), 403-409. https://doi.org/10.1016/j.tics.2009.06.003

Summerfield, J. J., Lepsien, J., Gitelman, D. R., Mesulam, M. M., \& Nobre, A. C. (2006). Orienting attention based on long-term memory experience. Neuron, 49(6), 905-916. https://doi.org/10.1016/j.neuron.2006.01.021

Summerfield, J. J., Rao, A., Garside, N., \& Nobre, A. C. (2011). Biasing perception by spatial long-term memory. Journal of Neuroscience, 31(42), 14952-14960. https://doi.org/10.1523/JNEUROSCI.5541-10.2011

Sutherland, M. R., \& Mather, M. (2012). Negative arousal amplifies the effects of saliency in short-term memory. Emotion, 12(6), 1367-1372. https://doi.org/10.1037/a0027860

Sutherland, M. R., \& Mather, M. (2015). Negative arousal amplifies the effects of saliency in short-term memory in older adults. Experimental Aging Research, 41(3), 259-271. https://doi.org/10.1080/0361073X.2015.1021644

Sutherland, M. R., \& Mather, M. (2018). Arousal (but not valence) amplifies the impact of salience. Cognition and Emotion, 32(3), 616-622. https://doi.org/10.1080/02699931.2017.1330189

Theeuwes, J. (2018). Visual selection: Usually fast and automatic; seldom slow and volitional. Journal of Cognition, 1(1), 1-15. https://doi.org/10.5334/joc.13

Theeuwes, J. (2019). Goal-driven, stimulus-driven and history-driven selection. Current Opinion in Psychology, 29, 97-101. https://doi.org/10.1016/j.copsyc.2018.12.024

Venables, L., \& Fairclough, S. H. (2009). The influence of performance feedback on goalsetting and mental effort regulation. Motivation and Emotion, 33(1), 63-74. https://doi.org/10.1007/s11031-008-9116-y

Vuilleumier, P., \& Pourtois, G. (2007). Distributed and interactive brain mechanisms during emotion face perception: Evidence from functional neuroimaging. Neuropsychologia, 45(1), 174-194. https://doi.org/10.1016/j.neuropsychologia.2006.06.003

Zeelenberg, R., \& Bocanegra, B. R. (2010). Auditory emotional cues enhance visual perception. Cognition, 115(1), 202-206. https://doi.org/10.1016/j.cognition.2009.12.004 
Table 1. Number of trials in each condition

\begin{tabular}{|c|c|c|c|c|c|c|c|c|c|}
\hline \multirow{3}{*}{$\begin{array}{l}\text { Session } 2 \\
\text { Conditions: } \\
\text { Session } 1 \\
\text { Conditions: } \\
\text { Block Number }\end{array}$} & \multicolumn{9}{|c|}{ Key Present trials } \\
\hline & \multicolumn{4}{|c|}{ Target Memory } & \multicolumn{5}{|c|}{ No-target Memory } \\
\hline & CS+ & CS- & UCS & Total & CS+ & CS- & UCS & Total & $\begin{array}{l}\text { Sub- } \\
\text { Total }\end{array}$ \\
\hline 1 & 4 & 5 & 2 & 11 & 5 & 4 & 2 & 11 & 22 \\
\hline 2 & 4 & 4 & 2 & 10 & 5 & 4 & 2 & 11 & 21 \\
\hline 3 & 5 & 4 & 2 & 11 & 4 & 5 & 1 & 10 & 21 \\
\hline Total & 13 & 13 & 6 & 32 & 14 & 13 & 5 & 32 & 64 \\
\hline Session 2 & \multicolumn{9}{|c|}{ Key Absent trials (catch trials) } \\
\hline Session 1 & \multirow{2}{*}{\multicolumn{4}{|c|}{ Target Memory }} & \multirow{2}{*}{\multicolumn{5}{|c|}{ No-target Memory }} \\
\hline Conditions: & & & & & & & & & \\
\hline Block Number & CS+ & CS- & UCS & Total & CS+ & CS- & UCS & Total & $\begin{array}{l}\text { Sub- } \\
\text { Total }\end{array}$ \\
\hline 1 & 2 & 3 & 0 & 5 & 2 & 2 & 1 & 5 & 10 \\
\hline 2 & 2 & 2 & 1 & 4 & 2 & 3 & 1 & 6 & 10 \\
\hline 3 & 3 & 2 & 1 & 6 & 2 & 2 & 1 & 5 & 11 \\
\hline Total & 7 & 7 & 2 & 16 & 6 & 7 & 3 & 16 & 32 \\
\hline Grand Total & & & & & & & & & 96 \\
\hline
\end{tabular}


Table 2. Means and standard deviations of accuracy (\%) in each condition

\begin{tabular}{lcc}
\hline Condition & Mean (\%) & SE \\
\hline CS+/Target Memory & 77.68 & 2.44 \\
CS+/No-target Memory & 50.70 & 2.44 \\
CS-/Target Memory & 72.78 & 2.44 \\
CS-/No-target Memory & 47.49 & 2.44 \\
\hline
\end{tabular}


A Training phase
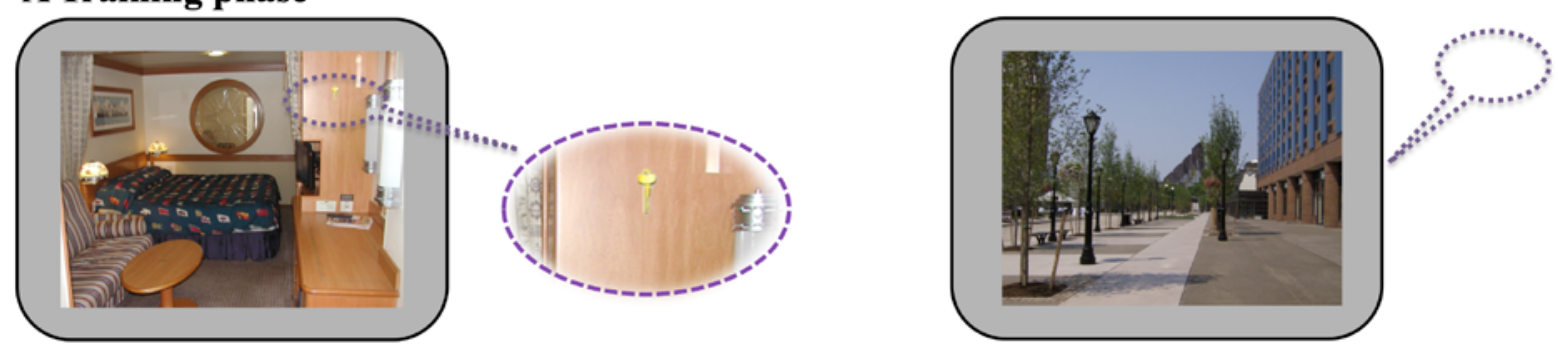

A1. Target memory trial

A2. No-target memory trial

\section{B Fear-conditioning}

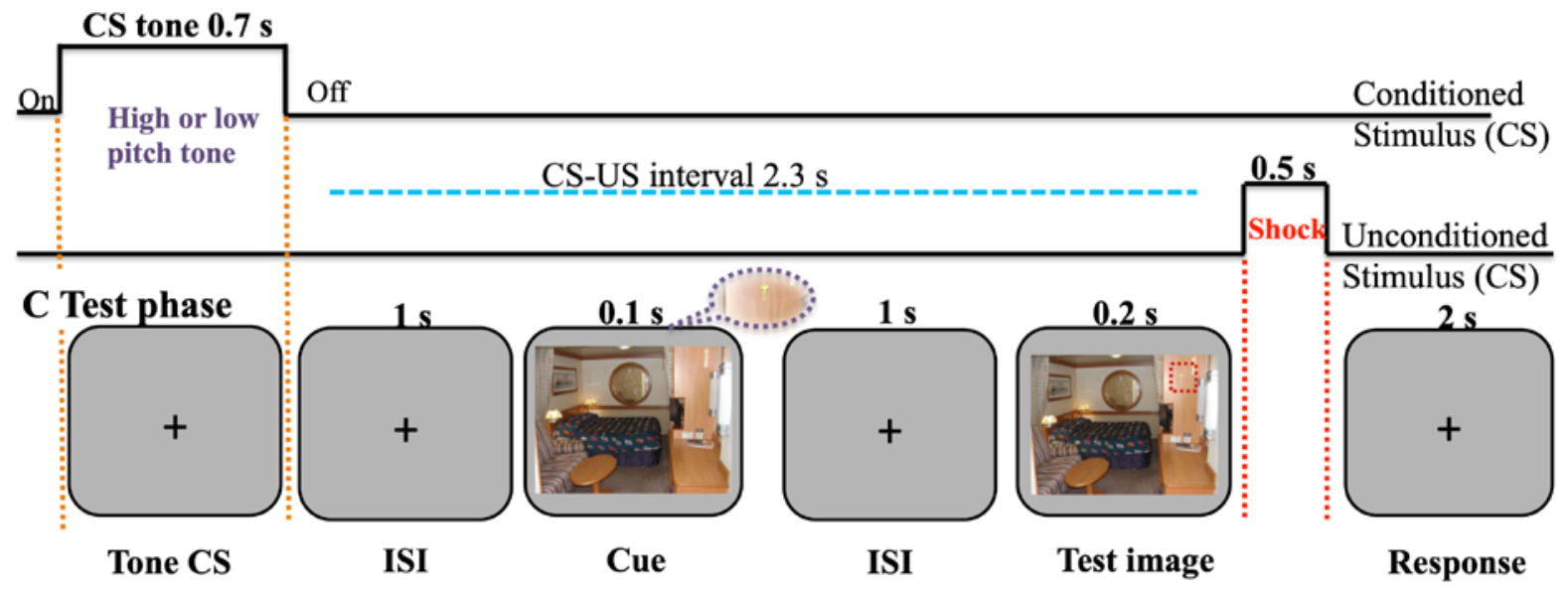

Figure 1. 
A

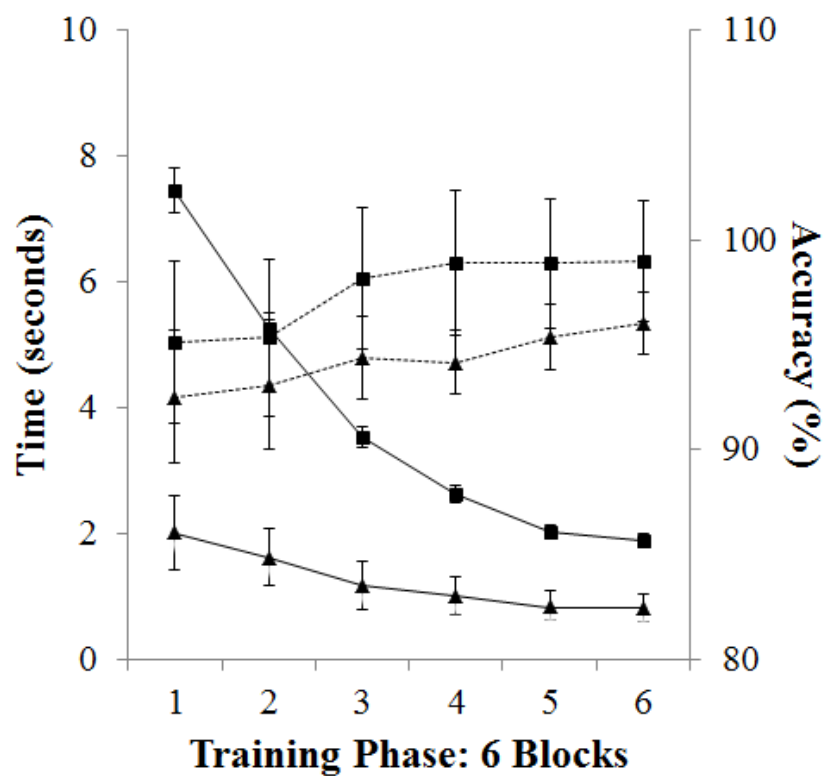

B

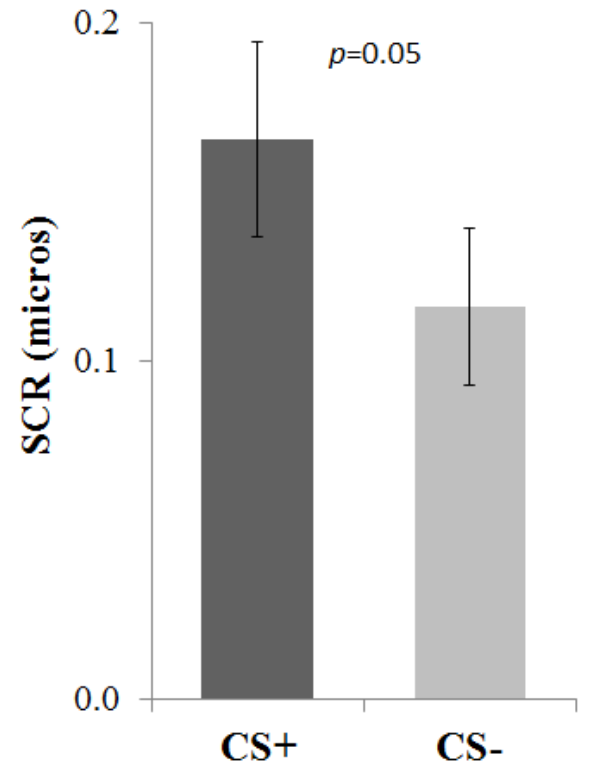

Figure 2. 


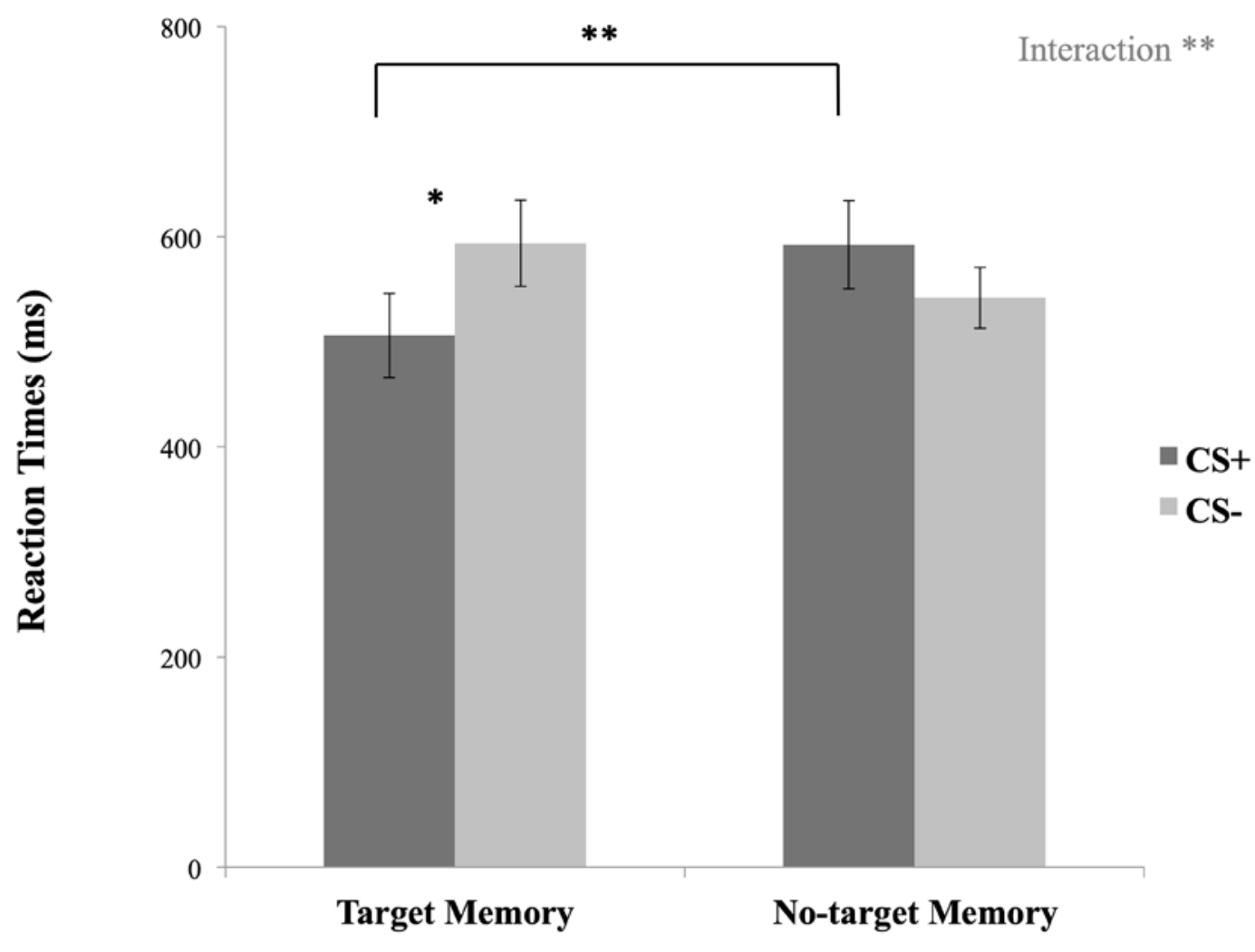

Figure 3. 


\section{Figure Captions:}

Figure 1. (A) Example stimuli used in the training phase, where participants were asked to indicate whether they detected the target (A1; the target memory condition) or not (A2; no-target memory condition). Schematic illustration of (B) a trial in the fear conditioning phase and (C) a trial in the test phase from the target memory condition. The interval between the conditioned stimulus (CS+) and electrical stimulation (shock) was identical between (B) the fear conditioning phase and (C) the test phase. The red square was drawn for presentation purposes only to show the yellow target key on the 'Test image'; participants saw all scenes without the square.

Figure 2. (A) Participants' performance improved over the six blocks in the training phase. The figure includes the reaction times (RTs; solid line) and the accuracy (dashed line) for the detection of the presence (squared line) and the absence (triangle line) of the target key within each scene. (B) Participants' SCR was higher in the CS+ condition compared to the CS- condition during the fear conditioning phase. Error bars represent the standard error of the mean (SEM).

$* * p<.01$

Figure 3. During the test phase, there was a significant interaction between arousal and memorysalience, such that CS+ facilitated the detection of the target key in the target memory condition. Error bars represent the standard error of the mean (SEM).

$* p<.01 * * p<.005$ 